1

\title{
Exploring metal resistance genes and mechanisms in copper
}

\author{
enriched metal ore metagenome
}

\author{
Esmaeil Forouzan $^{1}$, Ali Asghar Karkhane ${ }^{1}$, Bagher Yakhchali $^{1}$ *
}

1: Institute of Industrial and Environmental Biotechnology, National Institute of Genetic

Engineering and Biotechnology, Tehran, Iran.

*To whom correspondence should be addressed. bahar@nigeb.ac.ir

\section{Abstract}

Heavy metal pollution is a major global health challenge. In order to develop bioremediation solution for decontamination of environment from heavy metals one appropriate step is to investigate heavy metal resistance strategies used by microbial communities in the metal contaminated environments. The aim of the present study was to understand detailed mechanisms by which long time heavy metal (HM) exposed microbial community use to cope with excess of HMs. We exploited the Illumina high throughput metagenomic approach to examine taxonomical and functional diversity of copper enriched soil metagenome. Three enriched metagenomes were compared against 94 metagenomes derived from non- 
contaminated soils. Taxonomic composition analysis showed that phylogenetic profile of metal contaminated soils were enriched with $\gamma$-Proteobacteria. Comparison of functional profile of the two group reveled significant difference with potential role in HM resistance (HMR). Enriched SEED categories were "Membrane Transport", "Cell Wall and Capsule", "Stress Response", "Iron acquisition and metabolism" and "virulence and defense mechanisms". Raw metagenomic reads were assembled into scaffolds and predicted Open Reading Frames (ORFs) were searched against metal resistance gene database (BacMet). Based on enriched genes and gene categories and search of known HMR genes we concluded the microbial community cope with HM using at least 10 different mechanisms. Copper resistance genes were more abundant

29 in the metagenome relative to other metals and pumping metals out of the cell were more abundant relative to other HMR mechanism. Results of the present study could be very helpful in understanding of HMR mechanism used by microbial communities.

Keywords: metagenome, heavy metal, resistance mechanism, comparative metagenomics

As a result of industrialization and changes in the environment, Heavy metal (HM) pollution is a

35 major global health challenge, especially in developing countries ${ }^{1}$ and situation is worsening steadily ${ }^{2}$. Electroplating, alloy manufacturing, smelting, fertilizers, fungicides, pigment, plastic, refining processes, battery and mining are some of anthropogenic sources for heavy metals ${ }^{3,4}$. concentration. $\mathrm{Cu}$ could cause toxicity in the cell by replacing other metals used as a part of enzymes or other protein complexes, Reactive oxygen species (ROS) production, damage 
41 membrane integrity and denaturing of proteins $s^{5,6}$. Currently some approaches are available to

42 decontaminate HM contaminated aqueous solution, such as ion exchange resins and chemical

43 precipitation ${ }^{7}$ however these treatments are just efficient at high concentration of metals and

44 produce secondary contamination which require additional treatment ${ }^{8}$. HM concentration in

45 polluted water is often around $1 \mathrm{mg} / \mathrm{l}$ or even less and requires to be reduced to less than

46 acceptable limit. According to World Health Organization (WHO) heavy metal concentration

47 limits (in $\mathrm{mg} \backslash \mathrm{L}$ ) in drinking water are 0.010 for $\mathrm{Hg}$, $\mathrm{As}$, and $\mathrm{Pb}, 3.0$ for $\mathrm{Zn}$ and 0.003 for $\mathrm{Cd}^{9}$, This

48 task, i.e. reaching this very low level of metal concentration is not trivial. Physicochemical

49 procedure to remove HM from water are inefficient in this concentration and costly ${ }^{10}$.

50 Unlike organic contaminants, which can be degraded into benign molecules, metals are not

51 degradable in nature, hence remediation of all heavy metals are much more difficult than other

52 organic pollutants. Therefore, detoxification of metal-polluted environments has to rely on

53 their removal from contaminated sites. In order to develop bioremediation solution for

54 decontamination of environment from heavy metals one appropriate step is to study HMR

55 strategies used by microbial communities in the metal containing/contaminated environments.

56 There are some studies on this subject ${ }^{11-17}$. One of main source of heavy metal contamination

57 is metal ore mining and steel industry ${ }^{18}$ so metal resistance genes have to be more prevalent in

58 such metal contaminated sites relative to other less contaminated ones ${ }^{19}$.

59 The recent development of high-throughput DNA sequencing technologies, combined with the

60 development of bioinformatics and public databases, has drastically changed our perception of

61 microbial communities to different stresses. NGS technology made functional and taxonomical

62 profiling a routine task, which was very expensive, and time consuming before NGS ${ }^{20}$. Thanks 
63 to this technology nowadays even small biological laboratories are able to launch metagenomic

64 project and study environmental microbial community in great detail ${ }^{20}$. NGS has been used as

65 a powerful tool to explore microbial community response to different stresses including HM

66 contamination ${ }^{12}$. In a recent study on soil bacterial community structure in different iron

67 mining area ${ }^{21} 16 \mathrm{~s}$ amplicon sequencing reveals that HM polluted area had significant higher

68 bacterial alpha diversity than those from unpolluted area and contaminated area have

69 significantly different bacterial composition relative to non-contaminated sites. Some other

70 studies show different strategies used by different microbial communities in response to HM

71 contamination ${ }^{12}$, including HM precipitation by sulfides production, secretion of extracellular

72 phosphatases and metallophores, change in cell wall and capsule structure, modification of

73 lipopolysaccharides, periplasmic copper oxidation, modification of transporters,

74 methylation/volatilization, ROS detoxification and cytoplasmic metal accumulation ${ }^{12}$. As HM

75 bioremediation have to include metal removal from environment, among these strategies,

76 metal accumulation and HM precipitation have higher potential as a bioremediation method.

77 In the present study, we analyzed and compared $\mathrm{Cu}$ enriched soil metagenome to

78 metagenomes derived from non-contaminated soils and reveal the variations of the functional

79 and taxonomical between these two groups. Our findings could be useful in better

80 understanding of how microbial community cope with HM contamination and pave the way to

81 reach a bioremediation method to HM pollution.

82

\section{Methods}




\section{Sample collection and DNA extraction}

85 Three samples (S1, S2, and S3) were gathered from top surface soil $(5-15 \mathrm{~cm})$ from Hmyard iron ore, Semnan province, Iran on 2013-07-15. S1, S2, and S3 were sampled from different part of

87 Hmayard iron ore, an idle soil, a sample from near a tree grown in the ore and a sample from near an annual plant grown in the ore respectively. For each sample, approximately $500 \mathrm{~g}$ of

89 soil gathered and kept on ice in sterile plastic bags until reaching to the NIGEB lab. After

90 returning to the lab, $200 \mathrm{~g}$ of each sample soil stored at $-80 \mathrm{C}$ for subsequent molecular

91 analysis. Immediately the remaining soil were sieved through a 100-mesh to remove stones and

92 visible plant fragments and after enrichment DNA extraction was done according to Lee and

93 Hallam ${ }^{22}$. The remaining was air-dried at room temperature for one week and stored at $4{ }^{\circ} \mathrm{C}$ for

94 further chemical analysis.

\section{Soil physicochemical analysis}

96 In order to measure the soil HMs content we closely followed the methods reported by ${ }^{23}$.

97 Based on Akcay and coworkers, a fraction that contains exchangeable HM species is obtained

98 by the following procedure: each sample is extracted at room temperature for an hour with 10

$99 \mathrm{ml}$ of magnesium chloride solution (1M MgCl2 buffered at $\mathrm{pH} 7.0$ ) with continuous shaking. HM

100 concentration in the supernatant was measured after centrifugation at $3000 \mathrm{~g}$ using atomic

101 absorption spectrophotometry (Shimadzu AA670/G).

\section{Copper enrichment}

103 An enrichment process was used as described by Li et al ${ }^{24}$. It was assumed that copper 104 resistant microbial fraction of the community could be enriched under high copper 
concentration. Briefly two grams of fresh soil sample was mixed with $5 \mathrm{ml}$ sodium phosphate buffer $(0.1 \mathrm{M})$ and $50 \mu \mathrm{l}$ of the suspension spreaded over three LB agar plates containing copper $\left(200,400,800,1,000,1500,2000 \mathrm{mg} \mathrm{L}^{-1}\right)$. The LB agar plates were incubated at room for $72 \mathrm{~h}$. The results showed that on the $1,000 \mathrm{mg} \mathrm{L}^{-1}$ copper concentration 9 colonies were found and just 2 on $1500 \mathrm{mg} \mathrm{L}^{-1}$. According to this observation, 1,000 $\mathrm{mg} \mathrm{L}^{-1}$ was chosen as final concentration in enrichment process. For enrichment $5 \mathrm{gr}$ of soil was added to $5 \mathrm{ml}$ of enrichment solution (containing of sucrose and tryptose and starch, each $200 \mathrm{mg} \mathrm{L}^{-1}$ ) supplemented with $100 \mathrm{mg} \mathrm{L}^{-1}$ copper under sterile condition and incubated for 2 days room temperature and again enrichment solution was added. This cycle were repeated for 10 times in such a way that after the last cycle (20th day) the final concentration of copper in the soil was $1,000 \mathrm{mg} \mathrm{L}^{-1}$.

\section{Library construction and metagenomic sequencing}

After DNA extraction, quantity and quality of extracted DNA were measured using spectrophotometry (NanoDrop spectrophotometer) and agarose gel electrophoresis (1\%). DNA extraction was done in triplicate and pooled to minimize DNA extraction bias. Subsequently, DNA was sheared into fragments ( $400 \mathrm{bp})$ using ultrasonication. Paired-end library were built using the TruSeq2 Kit according to the manufacturer's instructions (http://www.illumina.com/). Resulting libraries were sequenced using Illumina HiSeq 2000 (Pair-end sequencing ( $2 * 100 \mathrm{bp}$ ) according to standard protocol (http://www.illumina.com/).

\section{Bioinformatics analysis}

The adaptors was removed and raw reads were trimmed based on two criteria, quality score $\geq 20$ and length $\geq 50$ bp using trimmomatic ${ }^{25}$. Clean paired reads were merged using FLASH ${ }^{26}$ 
127 and submitted to mg-rast sever for further analysis ${ }^{27}$. Reads data and the analysis results are

128 available with MG-RAST project number mgp83855. Merged reads were analyzed using MG-

129 RAST pipeline with default parameters. Clean reads were assembled using metaSPAdes

130 software ${ }^{28}$. MetaSPAdes was chosen because previous studies showed its good performance

131 relative to other assemblers ${ }^{29,30}$. Assembly was done with multiple $\mathrm{k}$-mer approach $(\mathbf{k}$-mers $=$

$13221,31,41,51$ and 61$)$. Genes were predicted in the resulting contigs using meta Gene Mark ${ }^{31}$.

133 For comparative part of the study a search was done for all shotgun soil metagenomes in MG-

134 RAST ${ }^{27}$ database. The resulted metagenomes were manually checked and a collection of 94

135 metagenomes from non-contaminated soil was obtained. Functional and taxonomical profile

136 for all soil metagenomes were retrieved using MG-RAST API ${ }^{32}$ and used as control group in

137 comparative analysis. Properties of metagenomes used in the study are available in Table S-

138 DatasetStat. Comparative metagenomics statistical test was done using STAMP ${ }^{33}$. For

139 comparison, white's non-parametric t-test was used with Benjamini-Hochberg as multiple test

140 error correction.

141 For HM resistance gene analysis, metal resistance genes database were downloaded from

142 BacMet database ${ }^{34}$. All of the predicted protein were searched against the database using

143 blastp. Blast results output were filtered based on three criteria, Evalue $\leq E-5$, coverage $\geq 90 \%$

144 and identity $\geq 70$. Best hit for each query was accounted for downstream analysis. Frequency of

145 resistant genes for each HM metal were calculated using self-written python script.

\section{Results}


Previous studies demonstrated Hmyard iron ore contains high level of copper and could be a source of metal contamination in the region (unpublished data). Metal concentration of iron ore soil for $\mathrm{Fe}, \mathrm{Cu}, \mathrm{Cr}$ and $\mathrm{Cd}$ were 919, 256, 11 and $0.5 \mathrm{mg} / \mathrm{kg}$ respectively. This shows copper and iron are in high concentration relative to other studied soils ${ }^{35,36}$ and could cause health problem to organisms living in there. Therefore we hypothesized microbial community of such community have to be resistant to HMs (at least to iron and copper).

\section{Quality control}

Clean paired reads were merged using flash ${ }^{26}$. Flash output was 24 million merged reads with average length of $160 \mathrm{bp}$. The merged reads were submitted to MG-RAST server for further analysis. Based on the DRISEE ${ }^{37}$ submitted reads error content was $\sim 1 \%$ and ambiguous character content was 0.003 . $28 \%$ of reads failed to pass MG-RAST QC criteria, however there were still enough reads to evaluate taxonomical and functional profile ${ }^{38}$. Detailed statistics are available in table S-DatasetStat. Dataset and resulted assembly properties are briefly overviewed in Table 1.

\section{Taxonomic composition}

Shanon diversity index for the enriched metagenome (EM) was 31, which is 16 fold smaller than that for average of control soils (Table S-DatasetStat), indicating that enrichment severely reduce population diversity. Rarefraction curve becomes almost flat to the right meaning that sequencing depth was enough to detect majority of the diversity available in the sample (figure S-Rarefraction). At domain level, EM contained 98\% Bacteria, 1.8\% Eukaryota, 0.01\% Viruses and negligible Archea. This means EM was enriched in bacteria and depleted in Eukaryota and 
Archea (figure S-domain). At phylum level, there are 13 taxa available in the EM with $0.01 \%$ or and depleted in actinobacteria. The Proteobacteria embraced mainly $\gamma$-Proteobacteria and Firmicutes mainly embraced bacilli. Compared to other non-contaminated environment $\gamma^{-}$

Proteobacteria are noticeably more prevalent in EM, in contrast some taxa such as $\alpha$ -

At the genus level, 15 genus obtained with relative abundance greater than $0.01 \%$ of the total

Staphylococcus, Arthrobacter and Pseudomonas consist more than $75 \%$ of total population with

Serratia alone consisting of more than $50 \%$ of the community (figure 1).

\section{Functional composition}

From the QC passed reads $86 \%$ were annotated as protein features, $10 \%$ as unknown protein

and $1 \%$ annotated as rRNA features. Figure 2 summarizes functional profile for level 1 of the

SEED subsystems. According to it, genes related to "Clustering-based subsystems" are the most abundant gene category in EM comprising $14 \%$ of total genes. "Carbohydrates" is the second prevailing gene category following by "Amino Acids and Derivatives". Photosynthesis were the mere frequency of gene categories does not make biological sense of the community. In order 
reveals interesting difference that points to mechanism underneath of HMR. We use available metagenomes derived from many (94 metagenomes, MG-RAST metagenome IDs are available in Table S-mgmID) different uncontaminated soils as control. Comparative study revealed that genes related to "iron acquisition and metabolism" were most enriched category in the EM relative to control metagenomes (Figure 3). "Membrane Transport", "Cell Wall and Capsule", "phosphorus metabolism", "potassium metabolism" and "Virulence Disease and Defense" are among enriched subsystems. "Protein Metabolism", "Cofactors, Vitamins, Prosthetic Groups, Pigments", and "Respiration" are among depleted subsystems in EM. Majority of the enriched categories are associated to known HMR processes. Comparative study for level 2 and 3 of SEED subsystem revealed difference in more details. Membrane transport encompass 9 202 subcategories at level 2 of the subsystems hierarchy including "Sym/Antiporters" and "ABC transporters", both of which are enriched in the EM. From "Iron acquisition and metabolism" group mainly genes related to siderophore production and secretion were enriched at level 2

205 (Table S-level2 and figure S-level2). "Quorum sensing and biofilm formation" is another enriched level 2 category with demonstrated role in HMR. Osmotic stress, periplasmic Stress,

207 Detoxification, Desiccation stress were enriched level 2 sub-categories of level 1 "Stress 208 Response" main category. "Resistance to antibiotics and toxic compounds" which is also enriched in EM have many subgroups related to HMR, such as Copper homeostasis, Mercury resistance operon and multidrug resistance efflux pumps. As expected, many of subcategories

211 bellow "Resistance to antibiotics and toxic compounds" are enriched in the EM but ironically 212 two group which we expected to be enriched were among the depleted categories, namely 213 "Cobalt-zinc-cadmium resistance" and "Zinc resistance". "Sugar alcohols" and "Proline and 4- 
214 hydroxyproline" are two enriched group with known function in abiotic stress. Isoprenoid/cell

215 wall biosynthesis is another enriched category with known function in heat and oxidative stress

$216{ }^{39}$ (Table-S-level2).

\section{HM resistant genes analysis}

Raw reads were assembled and genes in resulted metagenome were searched against HM resistance database ${ }^{34}$. This revealed available metal resistance genes in high resolution and their frequencies (Table 2, for more detailed Table refer to S-BacMet).

Majority of HM resistant genes are located on the chromosome (Table 2). According to the table, copper resistance genes were most abundant in the metagenome, consisting 131 genes

224 from 47 ortholog groups. From theses $\mathrm{Cu}$ resistance gene, CopA was more presented in the 225 metagenome followed by ctpG, which both encode heavy metal efflux P-type ATPases. For zinc 226 resistant genes top three most aboundant gene were baeS, yfeP and pitA, which encode two227 component regulatory system, Divalent metal cation transporter and Low-affinity inorganic 228 phosphate transporter respectively. An arsenic pump (arsB) is the most prevalent As resistant 229 protein and arsenate reductase ( $\operatorname{ars} \mathrm{C})$ being the second most prevalent resistance gene. 230 Regarding Fe resistance genes, 17 aconitase (acn) were detected in the metagenome and 7 231 divalent metal cation transporter. In addition, 5 member of the PmrA-PmrB two-component 232 system were present in the metagenome which are involved in controlling of bacterial response 233 to external $\mathrm{pH}$ and iron. PmrA-PmrB regulates genes that modify lipopolysaccharide. yhcN, yfeP 2347 and robA were main cadmium resistant genes available in the metagenome that encode 
235 proteins with unknown function, divalent metal cation transporter and transcriptional

236 activator. RobA can confer resistance to antibiotics, silver, mercury, and cadmium. Among

237 detected HM resistance genes, most frequently detected gene was ruvB. It encode for ATP-

238 dependent DNA helicase, which is involved in repairing DNA damage. ArsB, which encode

239 cytoplasmic arsenic pump protein, was the second most abundant HM resistant gene. RuvB and

240 arsB are followed by acn, copA, mgtA and G2alt. MgtA encode for magnesium-transporting

241 ATPase and G2alt has ATPase activity ${ }^{40}$.

242 Available HMR genes in EM revealed that different HMR gens are available in iron ore microbial

243 community, namely different family of metal extrusion pumps(such as P-type ATPases, RNDs,

244 MFS, Cation diffusion facilitators), metal chaperones, HM reductase, HM sensors, phosphatase,

245 siderophore reductase, transcription activator/repressor proteins and kinase proteins.

\section{Discussion}

\section{Taxonomic composition}

249 Based in our results, taxonomic composition of the EM was drastically different from that of a 250 typical soil, with a great enrichment of Proteobacteria mainly $\boldsymbol{\gamma}$-Proteobacteria. There are many

251 studies in which HM contaminated environments are taxonomically investigated. A previous

252 study concluded arsenic contamination changed taxonomic composition of mediterranean

253 marine sediments in favor of Proteobacteria ${ }^{38}$. Another study revealed that cadmium

254 contamination of soil resulted in increase of Proteobacteria (from 39 to 59\%) and decrease of

255 Acidobacteria (18 to $6 \%)^{41}$. Christopher and coworkers showed that metal-contaminated 
256 (mainly uranium contaminated) groundwater microbial community was composed primarily of

$257 \quad y$-Proteobacteria ${ }^{42}$. These three studies are in line with the present study. In addition, Li et al

258 studied copper stress effect on microbial community in activated sludge. They showed after

259 exposure to high copper stress, eukaryote portion of the community decreased significantly,

260 which is also in line with the present study. Li and coworkers shoed that copper stress resulted

261 in enrichment of Firmicutes, Chloroflexi and Spirochaetes; however, Proteobacteria were

262 depleted after copper enrichment. Both community, i.e. before and after copper enrichment

263 Proteobacteria were most abundant phyllum. ${ }^{24}$. Depletion of eukaryote portion of the

264 community after copper enrichment reported by $\mathrm{Li}$ is in accordance with the present study;

265 therefore, we could conclude that in a community metal resistance genes are mainly carried

266 out by prokaryotic part of the community. Gillan and coworkers evaluated long-term effect of

267 metal contamination on sediment and reported that taxonomical profile did not changed

268 significantly upon metal contamination at phylum level, but slight change was seen at genus

269 level, seven bacterial genera were significantly more abundant in metal contaminated $\operatorname{sites}^{13}$.

270 Very similar taxonomic composition between contaminated and uncontaminated sediment in

271 Gillan study could be due to low HM concentration and longtime of contamination, enabling

272 microbial community to adapt to new condition over long time. Pei et al compared soil sample

273 from chromium contaminated and uncontaminated region and revealed that Cr contaminated

274 sampling sites were taxonomically different from the uncontaminated ones. Particularly, the

275 relative abundances of Firmicutes and Bacteroidetes were higher while Actinobacteria was

276 lower in the contaminated group than uncontaminated group. In the Pei study most abundant

277 phyla present with the contaminated group were Proteobacteria, Bacteroidetes, and Firmicutes 
278 respectively ${ }^{43}$. Similar to Pei study, taxonomic profile of the EM were dominated by

279 Proteobacteria and Firmicutes but not Bacteroidetes.

280 There are different (sometimes even contrasting) results on the HM effect on taxonomical

281 composition of the soil. Some studies claim that HM contamination has insignificant effect on

282 taxonomical composition ${ }^{13,21}$, some reporting $\mathrm{HM}$ contamination results in enrichment of

283 Proteobacteria ${ }^{38,41,44}$ and in contrast some reports it results in depletion of Proteobacteria,

284 others reports it results in enrichment of Firmicutes ${ }^{24,43}$, Bacteroidetes $^{43}$ or other phyllums.

285 However even those studies reporting HM contamination leads to depletion of Proteobacteria,

286 simultaneously exclaimed that after HM contamination Proteobacteria still comprise main

287 portion of the community ${ }^{13,21}$. Therefore, we may conclude that HM contamination results in

288 different taxonomic change based on the evolutionary history of the community,

289 type/concentration of contaminated metal, physicochemical properties of environment and ...;

290 however, it seems that Proteobacteria are better adopted to HM contamination than other

291 bacterial phylum. This could be explained by Proteobacteria highly plastic genome, displaying

292 10-fold genome size variation and genome plasticity ${ }^{45}$. At lower taxonomic levels, the situation

293 is more complicated and hence generalization is more difficult.

295 Functional composition

296 Enrichment of "Cell Wall and Capsule" subsystem in EM revealed that microbial community

297 possibly cope with excess metal concentration by changing in their cell wall structure and

298 secretion of capsules. In line with our study, it was shown that the most prominent difference

299 between a HM contaminated and a less contaminated sediment is related to 'cell wall and 
300

301

302

303

304

305

306

307

308

309

310

311

312

313

314

315

316

317

318

319

320

321

capsule' subsystem which is more represented in contaminated sediment ${ }^{13}$. Therefore nonspecific binding of metals by extracytoplasmic part of the cell (outer membrane, cell wall, Slayer, lipopolysaccharide, extracellular polymeric substances) is present in the iron ore microbial community as a passive mechanism for metal exclusion. Deeper investigation showed that many genes related to "Cell Wall and Capsule" subsystem are enriched in the iom, such as peptidoglycan biosynthesis, lipopolysaccharide assembly, lipid A modifications and capsular polysaccharides Biosynthesis (Figure S-level3). This showed that several components of extracytoplasmic part of the cell are modified in response to HM. In addition, "Quorum sensing and biofilm formation" gene category is enriched in the EM. Generally biofilms are composed of extracellular polymeric substances and are capable of increasing HMR of attached communities $^{46}$. There is evidence that planktonic Pseudomonas aeruginosa is more susceptible to $\mathrm{HM}$ relative to it's biofilm form ${ }^{47}$. Therefore, we could conclude biofilm formation is also HMR strategy used by the microbial community. It has been demonstrate that quorum sensing increases resistance to osmotic, thermal and heavy metal stress ${ }^{48}$. Considering the iron ore metagenome was located in a hot semiarid region with high salinity, quorum sensing could be very useful to the bacterial community.

Enrichment of "Membrane Transport" category in the EM discloses another mechanism of HMR i.e. extrusion of extra HM ion out of cell. Many HM transporters from different classes are among the enriched genes including divalent metal transporter 1 (DMT1), cadmiumtransporting ATPase, RND efflux pumps, CzCD (a cation diffusion facilitator) and ECF transporters(Table S-enrichedGenes), all with known function in cell metal homeostasis ${ }^{49-51}$. Therefore, efflux mechanism is obviously present in the EM as a HM resistance method. 
Metagenomic studies have found high levels of $c z c D$ in an acid mine drainage metagenome and in a zinc contaminated sediment community ${ }^{13,52}$. In activated sludge of a Cu-contaminated

324 tannery wastewater copA was the most abundant of the $\mathrm{Cu}$ resistance systems ${ }^{53}$. It also has

325 been shown that in a cd contaminated soil most abundant pathway observed is ko02010, which 326 is assigned to $A B C$ transporters ${ }^{41}$.

327 Many of gene categories related to stress response, specifically oxidative stress response are 328 enriched in EM and this shows that "ROS scavenging and DNA reparation" being present in EM as another resistance mechanism. Some of the enriched genes are "Glutathione Redox cycle", "Universal stress response" and superoxide dismutase (Table S-enrichedGenes). Several reports

331 indicate that microbes exposed to high concentration of metals upregulate genes that are 332 involved in ROS scavenging, DNA reparation and hydrolysis of abnormally folded proteins ${ }^{54-56}$.

333 Such systems are indirect HMR mechanism that repair damages induced by metals. At the 334 community level genes involved in response to ROS were highly expressed in acid mine 335 drainage communities ${ }^{52,57}$.

336 Redox enzymes such as multicopper oxidase (CueO) and ferroxidase are also enriched in the EM 337 revealing another mechanism of HM tolerance, i.e. transforming metal ion to less harmful ones.

338 CueO expression confers copper tolerance in Escherichia coli ${ }^{58}$.Transformation of metal into 339 less harmful ones is an important resistance strategy and periplasmic copper oxidation were 340 shown to be more important mechanism than efflux in an extremely Cu-contaminated 341 activated sludge ${ }^{24}$.

342 Iron- bounded siderophores moves into the cell efficiently but siderophores bound to other HM 343 do not enter the cell readily and siderophore synthesis and secretion help the cell to tolerate 
$344 \mathrm{HM}$ in this way $^{12,59}$. According to the present state of knowledge siderophore mediated

345 resistance were not seen in microbial community and metallophore genes were not enriched in

346 the studied metal contaminated communities ${ }^{12}$. In contrast, the EM contain plenty of genes

347 related to siderophore synthesis and secretion and is significantly enriched relative to

348 uncontaminated soils(Table enrichedGenes), indicating that one of the main mechanism of

349 resistance by the community is siderophore synthesis and secretion.

350 Enrichment of "Potassium metabolism" category genes could be explained in two ways. First, as

351 the iron ore was located in a semiarid region with high salinity, enrichment of potassium

352 metabolism genes coud be a response to cope with high salinity of the environment. As shown

353 by many studies potassium metabolism has a close relationship with salinity stress tolerance

354 both in bacteria and plants ${ }^{60,61}$. Another explanation for the enrichment of potassium

355 metabolism in the EM could be positive tolerance against elevated dose of HMs, as in the case

356 for bacterial acidophiles. Acidophiles generating a chemiosmotic gradient by keeping up an

357 inside positive cytoplasmic transmembrane potential and hindering metal cation entering the

358 cytoplasm in this way ${ }^{62,63}$. We could hypothesize that potassium ion could be used for similar

359 strategy to cope with high level of salt and HMs simultaneously. Moreover some of metal efflux

360 pumps use potassium gradient of plasma membrane as driving force for metal extrusion and

361 potassium gradient may help the cell to cope with excess metal in this way too ${ }^{64}$. Enrichment

362 of glutathione-gated potassium efflux systems (Kef) that play role in protection against toxic

363 electrophilic compounds ${ }^{65}$ supports our hypothesis and makes it more interesting. Cytoplasmic

364 glutathione acts as a scavenger of electrophiles and its role in HM stress response is known ${ }^{66}$. 
365 Glutathione mediated activation of Kef systems integrates $\mathrm{K}^{+}$efflux and $\mathrm{H}+$ influx, and the consequent acidification of cytoplasm protects against electrophile-mediated stress ${ }^{65}$.

367 Amino acid metabolism related genes did not differ significantly between EM and control, but

368 genes related to metabolism of "Lysine, threonine, methionine, and cysteine" were enriched in

369 the EM (Tables S-level2 and S-level3). It is well known that sulfur containing molecule play key

370 role in response to HMs, such as glutathione, H2S, cysteine and phytochelatine ${ }^{67,68}$. Therefore,

371 enrichment of cysteine metabolism could be justified as a response to need for sulfur

372 containing molecules. Moreover, it has been shown that lysine catabolism in bacteria is related

373 to osmotic stress resistance ${ }^{69}$.

374 Analysis of available metal resistance genes in EM metagenome showed that resistant genes for

37524 metal were present in the metagenome even for those metals that were exist in the mine

376 soil in very low content, like silver, zinc and arsenic (Table S-BacMet). Enrichment allowed us to

377 detect many different resistance strategies that were used by the microbial community. Among

378 HMR mechanism, metal ion export seems to be more prevailed (Figure 2 and Figure S-level3);

379 meaning generally metal extrusion is the primary strategy for metal resistance in EM. Regarding

380 to copper, chromium and arsenic it seems that transformation to less harmful ion is an

381 important strategy beside extrusion. It has been reported that in a activated sludge genes

382 related to copper oxidase after copper-enrichment were more prevalent relative to other

383 copper resistant genes indicating that periplasmic copper oxidation is more important

384 mechanism than efflux ${ }^{24}$. This is in line with our study. Presence of extracellular phosphatase in

385 the enriched genes(Table S-BacMet) suggest phosphate mediated precipitation of HMs is used

386 by the microbial iron ore community against HMs. Presence of PmrG (encodes for 
387 lipopolysaccharide phosphatase) in EM metagenome support this claim. It has been shown that dephosphorylation of the Hep(II) phosphate in the core region of lipopolysaccharide by PmrG results in resistance against $\mathrm{Fe}^{3+}$ and $\mathrm{Al}^{3+70}$.

Based on available known HMR genes and comparative metagenomics we could concluded that 10 mechanism of HMR were available in the EM microbial community. Mechanism of HMR which were present in EM were: 1) metal extrusion, 2) cell wall modification, 3) secretion of exopolysaccharides, 4) modification of lipopolysaccharides, 5) detoxification of metals by

394 change in oxidation state using redox enzymes, 6) precipitation of metals by extracellular phosphatases, 7) decreasing HM import into the cytoplasm by using metallophores, 8) scavenging ROS produced by HM, 9) DNA reparation, and 10) detoxification of metals by complexing with metal-binding proteins.

\section{Conclusion}

Here we showed that high-throughput metagenomic sequencing of enriched metagenome could reveal resistance mechanism in high resolution. Bacteria cope with excess of HMs in their

401 environments in different ways. Bacterial mechanism of adaptation to high concentrations of

402 heavy metals have been shown in many cultivated species ${ }^{71}$. There are 22 known metal 403 resistance mechanism in isolated bacteria and concern different cell partitions, i.e. the 404 extracellular environment, outer membrane, periplasm, cytoplasmic membrane and the 405 cytoplasm $^{12}$. Of the 22 known metal-resistance mechanism, 14 were found in complex 406 communities $^{12}$. Based on the enriched genes and subsystems in the present study, we 407 demonstrate that microbial community of iron ore use at least 10 mechanisms of HMR. The 
408 results of our study expand the current knowledge of the mechanism of HM resistance in

409 microbial community of metal contaminated soil.

\section{Data Accessibility}

411 - Raw DNA reads are available on the MG-RAST database under the project number mgp83855.

412 - Final DNA sequence assembly: uploaded to GenBank (accession number wil be added soon)

413 - Sampling locations and related information: available online (on the MG-RAST database)

\section{Author Contributions Statement}

415 Esmaeil Forouzan: " wrote the main manuscript and prepared figures. All authors reviewed

416 the manuscript."

417 Ali Asghar Karkhane,

Bagher Yakhchali ${ }^{1 *}$

\section{Competing interests}

420 The authors declare no competing interests.

\section{$421 \quad$ References}

422 1. Chowdhury, S., Mazumder, M. A. J., Al-Attas, O. \& Husain, T. Heavy metals in drinking water:

423 Occurrences, implications, and future needs in developing countries. Sci. Total Environ. 569-570,

$424 \quad 476-488(2016)$.

425 2. Su, C., Jiang, L. \& Zhang, W. A review on heavy metal contamination in the soil worldwide: Situation,

426 impact and remediation techniques. Environ. Skept. Crit. (2014). 
3. Abdel -Aty, A. M., Ammar, N. S., Abdel Ghafar, H. H. \& Ali, R. K. Biosorption of cadmium and lead from aqueous solution by fresh water alga Anabaena sphaerica biomass. J. Adv. Res. 4, 367-374 (2013).

4. Roberts, T. L. Cadmium and Phosphorous Fertilizers: The Issues and the Science. Procedia Eng. 83, 52-59 (2014).

5. Liu, J., Qu, W. \& Kadiiska, M. B. Role of oxidative stress in cadmium toxicity and carcinogenesis. Toxicol. Appl. Pharmacol. 238, 209-214 (2009).

6. Nies, D. H. Microbial heavy-metal resistance. Appl. Microbiol. Biotechnol. 51, 730-750 (1999).

7. Aziz, H. A., Yusoff, M. S., Adlan, M. N., Adnan, N. H. \& Alias, S. Physico-chemical removal of iron from semi-aerobic landfill leachate by limestone filter. Waste Manag. 24, 353-358 (2004).

8. Feng, D., Aldrich, C. \& Tan, H. Treatment of acid mine water by use of heavy metal precipitation and ion exchange. Miner. Eng. 13, 623-642 (2000).

9. Cobbina, S. J., Duwiejuah, A. B., Quansah, R., Obiri, S. \& Bakobie, N. Comparative Assessment of $361-377$ (2011).

11. Chen, L.-X. et al. Shifts in microbial community composition and function in the acidification of a lead/zinc mine tailings. Environ. Microbiol. 15, 2431-2444 (2013). communities? Curr. Opin. Biotechnol. 38, 123-130 (2016). 
14. Hemme, C. L. et al. Lateral Gene Transfer in a Heavy Metal-Contaminated-Groundwater Microbial Community. mBio 7, e02234-02215 (2016).

15. Li, A.-D., Li, L.-G. \& Zhang, T. Exploring antibiotic resistance genes and metal resistance genes in plasmid metagenomes from wastewater treatment plants. Front. Microbiol. 6, 1025 (2015).

16. Luo, J. et al. Structural and functional variability in root-associated bacterial microbiomes of $\mathrm{Cd} / \mathrm{Zn}$ hyperaccumulator Sedum alfredii. Appl. Microbiol. Biotechnol. 101, 7961-7976 (2017).

17. Zhang, X., Niu, J., Liang, Y., Liu, X. \& Yin, H. Metagenome-scale analysis yields insights into the structure and function of microbial communities in a copper bioleaching heap. BMC Genet. 17, 21 (2016).

18. Li, Z., Ma, Z., van der Kuijp, T. J., Yuan, Z. \& Huang, L. A review of soil heavy metal pollution from mines in China: Pollution and health risk assessment. Sci. Total Environ. 468-469, 843-853 (2014).

19. Zhuang, P., McBride, M. B., Xia, H., Li, N. \& Li, Z. Health risk from heavy metals via consumption of food crops in the vicinity of Dabaoshan mine, South China. Sci. Total Environ. 407, 1551-1561 (2009).

20. Boughner, L. A. \& Singh, P. Microbial Ecology: Where are we now? Postdoc J. J. Postdr. Res. Postdr. Aff. 4, 3-17 (2016).

21. Hong, C., Si, Y., Xing, Y. \& Li, Y. Illumina MiSeq sequencing investigation on the contrasting soil bacterial community structures in different iron mining areas. Environ. Sci. Pollut. Res. Int. 22,

22. Lee, S. \& Hallam, S. J. Extraction of high molecular weight genomic DNA from soils and sediments. J. Vis. Exp. JoVE (2009). doi:10.3791/1569 and Gediz river sediments. Water Res. 37, 813-822 (2003). 
24. Li, L.-G., Cai, L., Zhang, X.-X. \& Zhang, T. Potentially novel copper resistance genes in copperenriched activated sludge revealed by metagenomic analysis. Appl. Microbiol. Biotechnol. 98, $10255-10266$ (2014).

25. Bolger, A. M., Lohse, M. \& Usadel, B. Trimmomatic: a flexible trimmer for Illumina sequence data. Bioinforma. Oxf. Engl. 30, 2114-2120 (2014).

26. Magoč, T. \& Salzberg, S. L. FLASH: fast length adjustment of short reads to improve genome assemblies. Bioinforma. Oxf. Engl. 27, 2957-2963 (2011).

27. Keegan, K. P., Glass, E. M. \& Meyer, F. MG-RAST, a Metagenomics Service for Analysis of Microbial Community Structure and Function. Methods Mol. Biol. Clifton NJ 1399, 207-233 (2016).

28. Nurk, S., Meleshko, D., Korobeynikov, A. \& Pevzner, P. A. metaSPAdes: a new versatile metagenomic assembler. Genome Res. 27, 824-834 (2017).

29. Forouzan, E., Maleki, M. S. M., Karkhane, A. A. \& Yakhchali, B. Evaluation of nine popular de novo assemblers in microbial genome assembly. J. Microbiol. Methods (2017). doi:10.1016/j.mimet.2017.09.008

30. Forouzan, E., Shariati, P., Maleki, M. S. M., Karkhane, A. A. \& Yakhchali, B. Practical evaluation of 11 de novo assemblers in metagenome assembly. J. Microbiol. Methods (2018). doi:10.1016/j.mimet.2018.06.007

31. Zhu, W., Lomsadze, A. \& Borodovsky, M. Ab initio gene identification in metagenomic sequences. Nucleic Acids Res. 38, e132-e132 (2010).

32. Wilke, A. et al. A RESTful API for accessing microbial community data for MG-RAST. PLoS Comput. Biol. 11, e1004008 (2015).

33. Parks, D. H., Tyson, G. W., Hugenholtz, P. \& Beiko, R. G. STAMP: statistical analysis of taxonomic and functional profiles. Bioinformatics 30, 3123-3124 (2014). 
34. Pal, C., Bengtsson-Palme, J., Rensing, C., Kristiansson, E. \& Larsson, D. G. J. BacMet: antibacterial biocide and metal resistance genes database. Nucleic Acids Res. 42, D737-D743 (2014).

35. Qing, X., Yutong, Z. \& Shenggao, L. Assessment of heavy metal pollution and human health risk in urban soils of steel industrial city (Anshan), Liaoning, Northeast China. Ecotoxicol. Environ. Saf. 120, 377-385 (2015).

36. Jaffar, S. T. A. et al. The Extent of Heavy Metal Pollution and Their Potential Health Risk in Topsoils of the Massively Urbanized District of Shanghai. Arch. Environ. Contam. Toxicol. 73, 362-376 (2017).

37. Keegan, K. P. et al. A Platform-Independent Method for Detecting Errors in Metagenomic Sequencing Data: DRISEE. PLOS Comput. Biol. 8, e1002541 (2012).

38. Plewniak, F. et al. Metagenomic insights into microbial metabolism affecting arsenic dispersion in Mediterranean marine sediments. Mol. Ecol. 22, 4870-4883 (2013).

39. Vickers, C. E. et al. Isoprene synthesis protects transgenic tobacco plants from oxidative stress. Plant Cell Environ. 32, 520-531 (2009).

40. Nakajima, H., Kobayashi, K., Kobayashi, M., Asako, H. \& Aono, R. Overexpression of the robA gene increases organic solvent tolerance and multiple antibiotic and heavy metal ion resistance in Escherichia coli. Appl. Environ. Microbiol. 61, 2302-2307 (1995).

41. Feng, G. et al. Metagenomic analysis of microbial community and function involved in cdcontaminated soil., Metagenomic analysis of microbial community and function involved in cdcontaminated soil. BMC Microbiol. BMC Microbiol. 18, 18, 11-11 (2018). groundwater microbial community. ISME J. 4, 660-672 (2010).

518 43. Pei, Y., Yu, Z., Ji, J., Khan, A. \& Li, X. Microbial Community Structure and Function Indicate the 519 Severity of Chromium Contamination of the Yellow River. Front. Microbiol. 9, (2018). 
44. Li, X. et al. Response of soil microbial communities and microbial interactions to long-term heavy metal contamination. Environ. Pollut. Barking Essex 1987 231, 908-917 (2017).

45. Boussau, B., Karlberg, E. O., Frank, A. C., Legault, B.-A. \& Andersson, S. G. E. Computational inference of scenarios for $\alpha$-proteobacterial genome evolution. Proc. Natl. Acad. Sci. 101, 97229727 (2004).

46. Harrison, J. J., Ceri, H. \& Turner, R. J. Multimetal resistance and tolerance in microbial biofilms. Nat. Rev. Microbiol. 5, 928-938 (2007).

47. Teitzel, G. M. \& Parsek, M. R. Heavy Metal Resistance of Biofilm and Planktonic Pseudomonas aeruginosa. Appl. Environ. Microbiol. 69, 2313-2320 (2003).

48. García-Contreras, R. et al. Quorum sensing enhancement of the stress response promotes resistance to quorum quenching and prevents social cheating. ISME J. 9, 115-125 (2015).

49. Garrick, M. D. et al. DMT1: Which metals does it transport? Biol. Res. 39, (2006).

50. Herrou, J. et al. Conserved ABC Transport System Regulated by the General Stress Response Pathways of Alpha- and Gammaproteobacteria. J. Bacteriol. 199, e00746-16 (2017).

51. Rodionov, D. A., Hebbeln, P., Gelfand, M. S. \& Eitinger, T. Comparative and Functional Genomic Analysis of Prokaryotic Nickel and Cobalt Uptake Transporters: Evidence for a Novel Group of ATPBinding Cassette Transporters. J. Bacteriol. 188, 317-327 (2006).

52. Chen, L. et al. Comparative metagenomic and metatranscriptomic analyses of microbial communities in acid mine drainage. ISME J. 9, 1579-1592 (2015).

53. Jia, S. et al. Metagenomic analysis of cadmium and copper resistance genes in activated sludge of a tannery wastewater treatment plant. J. Environ. Biol. 34, 375-380 (2013).

54. Miller, C. D. et al. Copper and cadmium: responses in Pseudomonas putida KT2440. Lett. Appl. Microbiol. 49, 775-783 (2009). 
55. Poirier, I., Hammann, P., Kuhn, L. \& Bertrand, M. Strategies developed by the marine bacterium Pseudomonas fluorescens BA3SM1 to resist metals: A proteome analysis. Aquat. Toxicol. Amst. Neth. 128-129, 215-232 (2013).

56. Zhang, X. et al. Global transcriptome analysis of hexavalent chromium stress responses in Staphylococcus aureus LZ-01. Ecotoxicol. Lond. Engl. 23, 1534-1545 (2014).

57. Bertin, P. N. et al. Metabolic diversity among main microorganisms inside an arsenic-rich ecosystem revealed by meta- and proteo-genomics. ISME J. 5, 1735-1747 (2011).

58. Grass, G. \& Rensing, C. CueO Is a Multi-copper Oxidase That Confers Copper Tolerance in Escherichia coli. Biochem. Biophys. Res. Commun. 286, 902-908 (2001).

59. Neilands, J. B. Siderophores: structure and function of microbial iron transport compounds. J. Biol. Chem. 270, 26723-26726 (1995).

60. Wang, M., Zheng, Q., Shen, Q. \& Guo, S. The Critical Role of Potassium in Plant Stress Response. Int. J. Mol. Sci. 14, 7370-7390 (2013).

61. Yaakop, A. S. et al. Characterization of the mechanism of prolonged adaptation to osmotic stress of Jeotgalibacillus malaysiensis via genome and transcriptome sequencing analyses. Sci. Rep. 6, 33660 (2016).

62. Dopson, M., Ossandon, F. J., Lövgren, L. \& Holmes, D. S. Metal resistance or tolerance? Acidophiles confront high metal loads via both abiotic and biotic mechanisms. Front. Microbiol. 5, 157 (2014).

63. Wheaton, G., Counts, J., Mukherjee, A., Kruh, J. \& Kelly, R. The Confluence of Heavy Metal Biooxidation and Heavy Metal Resistance: Implications for Bioleaching by Extreme Thermoacidophiles. Minerals 5, 397-451 (2015).

64. Nies, D. H. Efflux-mediated heavy metal resistance in prokaryotes. FEMS Microbiol. Rev. 27, 313339 
65. Roosild, T. P. et al. Mechanism of ligand-gated potassium efflux in bacterial pathogens. Proc. Natl. Acad. Sci. U. S. A. 107, 19784-19789 (2010).

66. Jozefczak, M., Remans, T., Vangronsveld, J. \& Cuypers, A. Glutathione Is a Key Player in MetalInduced Oxidative Stress Defenses. Int. J. Mol. Sci. 13, 3145-3175 (2012).

67. Giles, N. M. et al. Metal and Redox Modulation of Cysteine Protein Function. Chem. Biol. 10, 677693 (2003).

68. Jia, H. et al. Hydrogen sulfide - cysteine cycle system enhances cadmium tolerance through alleviating cadmium-induced oxidative stress and ion toxicity in Arabidopsis roots. Sci. Rep. 6, 39702 (2016).

69. Neshich, I. A., Kiyota, E. \& Arruda, P. Genome-wide analysis of lysine catabolism in bacteria reveals new connections with osmotic stress resistance. ISME J. 7, 2400-2410 (2013).

70. Nishino, K. et al. Identification of the lipopolysaccharide modifications controlled by the Salmonella PmrA/PmrB system mediating resistance to Fe(III) and Al(III). Mol. Microbiol. 61, 645-654 (2006).

71. Lemire, J. A., Harrison, J. J. \& Turner, R. J. Antimicrobial activity of metals: mechanisms, molecular targets and applications. Nat. Rev. Microbiol. 11, 371-384 (2013).

\section{Figure legend:}

Figure 1: Taxonomic composition of copper enriched metagenome at phylum and genus level. Figure 2: SEED subsystem (level1) distribution differences between the HMC (heavy metal contaminated) and control (HM uncontaminated) soil metagenomes.

Figure 3: Functional comparative study of enriched heavy metal contaminated (HMC) soils with metagenomes derived from non-contaminated soils.

\section{Supplementary information.}

Table S-PCA: principle component analysis for soil metagenomes used in this study at the level 1 of SEED subsystems.

Table S-BacMet: Available metal resistance genes in HMC soil metagenome, frequencies for each resistance categories and location of the genes.

Table S-DatasetStat: properties for metagenomes used in the study. 
595 Table S-enrichedGenes: Enriched and depleted genes in the copper enriched metagenome

596 Table S-level2: Enriched and depleted level2 SEED subsystems in the copper enriched

597 metagenome

598 Table S-level3: Enriched and depleted level3 SEED subsystems in the copper enriched

599 metagenome

600 Table S-mgmID: 94 IDs for shotgun metagenomes downloaded from MG-RAST used as control

601 for comparative study.

602 Figure S-class: taxonomic comparison of the copper enriched metagenome and the control

603 group at class level.

604 Figure S-domain: taxonomic comparison of the copper enriched metagenome and the control 605 group at domain level.

606 Figure S-level2: functional comparison of the copper enriched metagenome and the control 607 group at level 2 of the SEED subsystem.

608 Figure S-level3: functional comparison of the copper enriched metagenome and the control 609 group at level 3 of the SEED subsystem.

610 Figure S-Rarefraction: Rarefraction for the copper enriched metagenome

611

612

613

Table 1: Overview of dataset and resulted assembly

\begin{tabular}{|l|l|}
\hline Category & metagenome \\
\hline Number of contigs(larger than $500 \mathrm{bp})$ & 9,347 \\
\hline Total length (bp) & $152,316,274$ \\
\hline N50* (bp) & 56,486 \\
\hline L50* (bp) & 500 \\
\hline Number of N's per 100 kbp & 16 \\
\hline Number of predicted genes & 136,177 \\
\hline contig mean length (bp) & 9,578 \\
\hline raw reads & $227,127,502$ \\
\hline Clean reads & $181,596,102$ \\
\hline raw data (bp) & $22,939,877,702$ \\
\hline clean data (bp) & $18,341,206,302$ \\
\hline
\end{tabular}

* N50 is the length for which the collection of all contigs of that length or longer covers at least 
bioRxiv preprint doi: https://doi.org/10.1101/2020.07.02.184564; this version posted July 2, 2020. The copyright holder for this preprint (which was not certified by peer review) is the author/funder, who has granted bioRxiv a license to display the preprint in perpetuity. It is made available under aCC-BY-ND 4.0 International license.

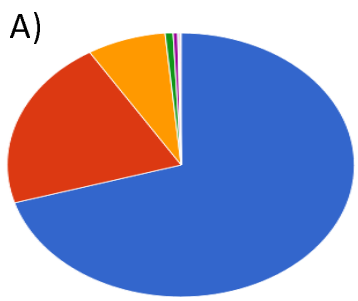

B)

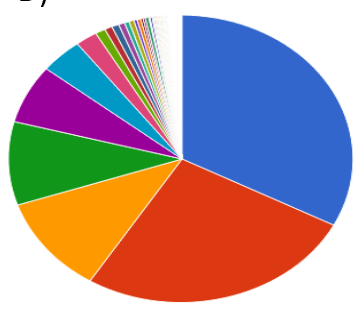

- Proteobacteria - $2,981,384(70.32 \%)$

- Firmicutes $-886,011(20.90 \%)$

Actinobacteria - 310,235 (7.32\%)

Basidiamycota - 30,872 (0.73\%)

- Ascomycota $-21,646(0.51 \%)$

Chordata - 3,921 $(0.09 \%)$

Streptophyta $-2,032(0.05 \%)$

Bacteroidetes - $489(0.01 \%)$

- Cyanobacteria - $360(0.01 \%)$

unclassified (derived from Viruses) - 334 (0.01\%)

Fusobacteria - $314(0.01 \%)$

Chrysiogenetes - $299(0.01 \%)$

Chlorophyta - $229(0.01 \%)$

- Tenericutes - $209(0.00 \%)$

- Serratia - $671,534(32.65 \%)$

Bacillus - 540,159 (26,26\%)

Staphylococcus - 223,466 (10.87\%)

Arthrobacter - 191,531 (9.31\%)

- Arthrobacter - $191,531(9.31 \%)$

Enterococcus - 82,590 (4.02\%)

- Brevibacterium - 41,752 (2.03\%)

- Yersinia - $18,853(0.92 \%)$

Klebsiella - 13,961 (0.68\%)

Filobasidiella $-13,901(0.68 \%)$

- Pantaea - $11,936(0.58 \%)$

Burkholderia $-9,705(0.47 \%)$

Erwinia - $8,360(0.41 \%)$

Escherichia - 7,956 (0.39\%)

617 Figure 1: Taxonomic composition of copper enriched metagenome at phylum (A) and genus (B) level. 


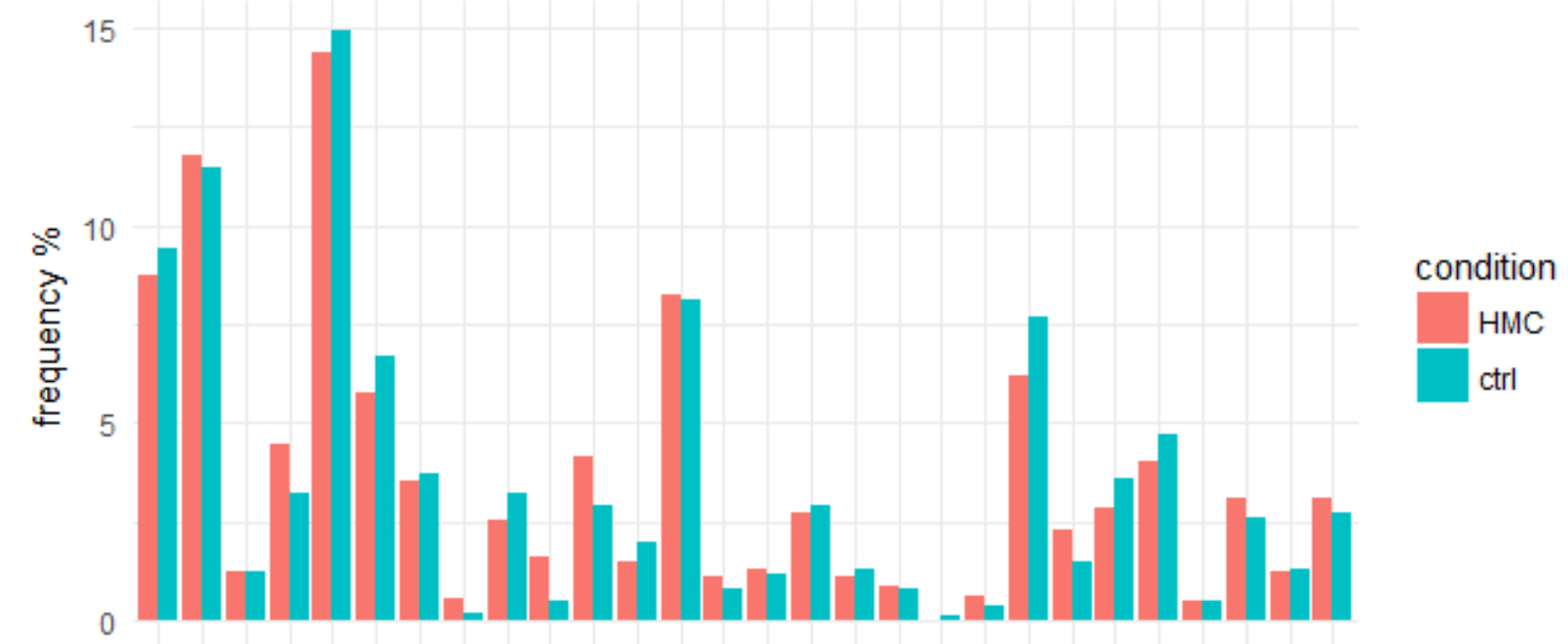

Figure 2: SEED subsystem (level1) distribution differences between the HMC (heavy metal contaminated) and control (HM uncontaminated) soil metagenomes based on MG-RAST annotation. The cutoff parameters for read annotation were default of MG-RAST (identity $\geq 60 \%$ and e-value $\leq 1 \times 10-5$ ). Comparison for level2, 3 and function level are available as supporting information (Figures and Tables names with prefix S-level2, S-level3 and S-Function). 
bioRxiv preprint doi: https://doi.org/10.1101/2020.07.02.184564; this version posted July 2, 2020. The copyright holder for this preprint (which was not certified by peer review) is the author/funder, who has granted bioRxiv a license to display the preprint in perpetuity. It is made available under aCC-BY-ND 4.0 International license.

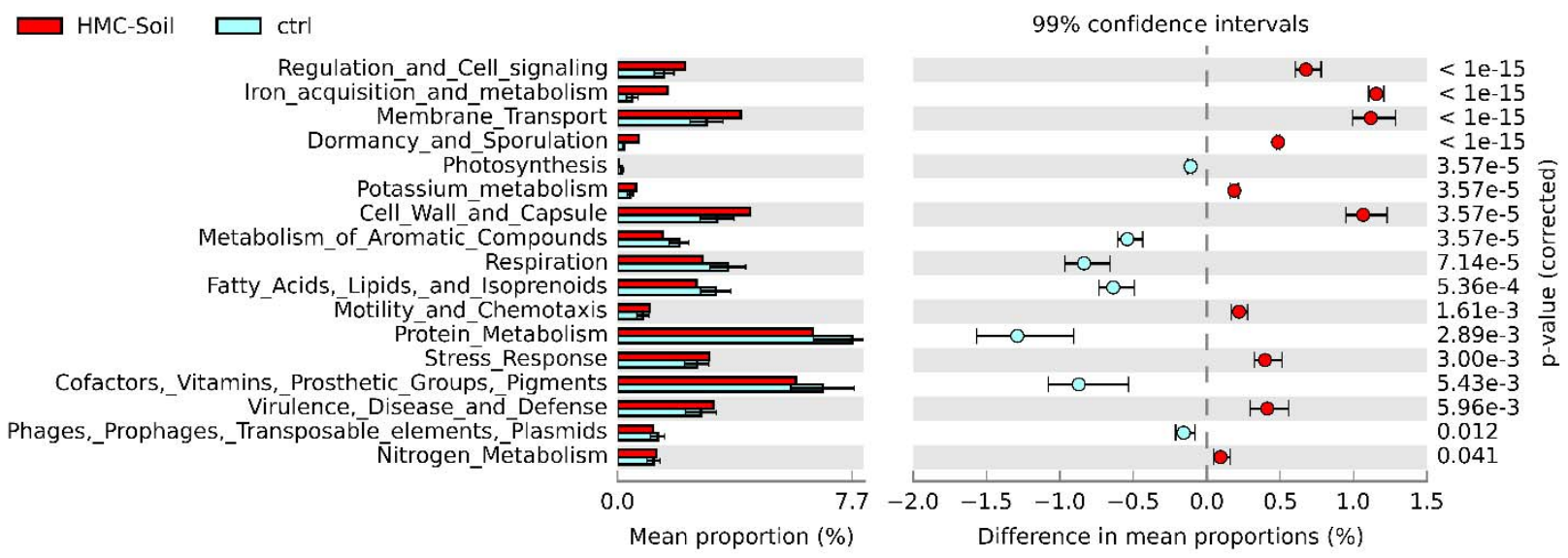

633 Figure 3: Functional comparative study of enriched heavy metal contaminated (HMC) soils with metagenomes derived from non-contaminated soils. Enriched gene categories in the EM metagenome has a positive difference between proportions (red circles), while depleted gene categories in the EM metagenome has a negative difference between proportions (blue circles). Bars on the left represent the proportion of each gene categories in the data. $P$ value of $\geq 0.05$ were considered significant. 
Table 2: Available metal resistance genes in HMC soil metagenome, frequencies for each resistance categories and location of the genes. More information is available in the table S-

649 BacMet.

\begin{tabular}{|c|c|c|c|}
\hline Resistance & $\begin{array}{l}\text { chromosome } \\
\text { gene } \\
\text { frquency }\end{array}$ & $\begin{array}{l}\text { plasmid } \\
\text { gene } \\
\text { frquency }\end{array}$ & Gene symbol and its frquency \\
\hline Copper (Cu) & 108 & 23 & $\begin{array}{l}\operatorname{copA} 12 ; \operatorname{ctpG} 8 ; \operatorname{cusA} / \text { ybdE } 7 ; \text { bhsA/ycfR/comC } 7 ; \operatorname{copB} 7 ; \\
\text { copZ } 6 ; \text { dsbC } 5 ; \text { yfmO } 5 ; \operatorname{cutC} 5 ; \text { cutE/Int } 5 ; \operatorname{cutA} 5 ; \operatorname{copR} 4 ; \\
\text { csoR } 4 ; \text { cueR/ybbl } 4 ; \text { zupT/ygiE } 4 \ldots\end{array}$ \\
\hline Zinc $(Z n)$ & 94 & 12 & $\begin{array}{l}\text { baes } 8 \text {; mntH/yfeP } 7 \text {; pitA } 7 \text {; znuB/yebl } 7 \text {; znuA/yebL } 6 \text {; } \\
\text { znuC/yebM } 5 \text {; baeR } 5 \text {; dsbA } 5 \text {; zur/yjbK } 5 \text {; zntR/yhdM } 4 \text {; } \\
\text { zraR/hydH } 4 \text {; zinT/yodA } 4 \text {; zntA/yhhO } 4 \ldots\end{array}$ \\
\hline Arsenic (As) & 59 & 38 & $\begin{array}{l}\operatorname{arsB} 18 ; \operatorname{arsC} 16 ; \text { pstB } 13 ; \operatorname{arsR} 11 ; \operatorname{arsT} 8 ; \operatorname{glpF} 7 ; \text { pstA } 7 ; \\
\text { pstC } 6 ; \text { pstS } 5 ; \operatorname{arsA} 3 ; \operatorname{arsH} 2 ; \operatorname{arsD} 1\end{array}$ \\
\hline Iron (Fe) & 83 & 10 & $\begin{array}{l}\text { acn } 17 \text {; mntH/yfeP } 7 \text {; pmrA } 5 \text {; pmrB } 5 \text {; fetB/ybbM } 5 \text {; ideR } 4 \\
\text {; sitA } 4 \text {; zupT/ygiE } 4 \text {; yqjH } 4 \text {; yfeB } 4 \text {; pmrC } 4 \text {; fetA/ybbL } 3 \ldots\end{array}$ \\
\hline Cadmium (Cd) & 52 & 19 & $\begin{array}{l}\text { yhcN } 8 ; \text { mntH/yfeP } 7 \text {; robA } 6 \text {; ygiw } 5 \text {; dsbB } 5 \text {; dsbA } 5 \text {; } \\
\text { cadD } 4 \text {; czcR } 4 \text {; zupT/ygiE } 4 \ldots\end{array}$ \\
\hline Cobalt (Co) & 55 & 7 & $\begin{array}{l}\text { mgtA } 10 ; \text { mntH/yfeP } 7 ; \operatorname{corD} 6 ; \operatorname{corC} 6 ; \text { fecE } 5 ; \operatorname{czcR} 4 ; \text { corA } \\
4 ; \text { zupT/ygiE } 4 ; \text { rcnR/yohL } 3 ; \operatorname{cmtR} 2 \ldots\end{array}$ \\
\hline Nickel (Ni) & 56 & 3 & $\begin{array}{l}\text { nikA } 5 \text {; fecE } 5 \text {; nikD } 4 \text {; corA } 4 \text {; zupT/ygiE } 4 \text {; nikB } 4 \text {; yqjH } 4 \text {; } \\
\text { ncrA } 3 \text {; nikR } 3 \text {; nikC } 3 ; \operatorname{rcnR} / \text { yohL } 3 \text {; nikE } 3 \ldots\end{array}$ \\
\hline Chromium (Cr) & 43 & 0 & $\begin{array}{l}\text { ruvB } 23 \text {; yieF } 6 \text {; recG } 5 \text {; chrR } 3 \text {; nfsA } 3 ; \text { mdrL/yfmo } 2 \text {; oscA } \\
1 . .\end{array}$ \\
\hline Manganese (Mn) & 32 & 10 & $\begin{array}{l}\text { mntH/yfeP } 7 \text {; mntP/yebN } 6 ; \text { mntR } 4 \text {; corA } 4 \text {; yfeB } 4 \text {; sitA } 4 \text {; } \\
\text { sitB } 2 \text {; sitC } 2 \text {; yfeD } 2 \text {; yfeC } 2 \ldots\end{array}$ \\
\hline Tellurium (Te) & 37 & 5 & ruvB 19 ; pitA 7 ; recG 5 ; terD 4 ; tehB 4 ; tehA $2 \ldots$ \\
\hline Antimony (Sb) & 7 & 31 & $\operatorname{arsB} 12 ; \operatorname{arsR} 8 ; \operatorname{arsC} 7 ; \operatorname{glpF} 7 ; \operatorname{arsA} 3 ; \operatorname{arsD} 1 \ldots$ \\
\hline Selenium (Se) & 36 & 0 & ruvB $19 ;$ sodA 7 ; sodB 5 ; recG $5 \ldots$ \\
\hline Magnesium (Mg) & 36 & 0 & mgtA $10 ;$ mntP/yebN $6 ; \operatorname{corD} 6 ; \operatorname{cor} C 6 ; \operatorname{mntR} 4 ; \operatorname{cor} A 4 \ldots$ \\
\hline Silver (Ag) & 13 & 19 & $\begin{array}{l}\text { cusA/ybdE } 7 \text {; robA } 6 \text {; copB } 5 \text {; pcoE } 2 \text {; cusR/ylcA } 1 \text {; cueA } 1 \text {; } \\
\text { silP } 1 \text {; silB } 1 \ldots\end{array}$ \\
\hline Tungsten (W) & 30 & 0 & $\begin{array}{l}\text { baes } 8 ; \text { baeR } 5 ; \bmod C 4 ; \bmod A 4 ; \bmod E 4 ; \operatorname{modB} 4 ; \operatorname{tupC} 1 \\
\ldots\end{array}$ \\
\hline Mercury (Hg) & 18 & 7 & $\begin{array}{l}\text { robA } 6 \text {; dsbB } 5 \text {; dsbA } 5 \text {; merT } 2 \text {; merA } 2 \text {; PA0320 } 2 \text {; merB } \\
1 \ldots .\end{array}$ \\
\hline Molybdenum (Mo) & 22 & 0 & yieF $6 ; \bmod C 4 ; \bmod A 4 ; \bmod E 4 ; \operatorname{modB} 4 \ldots$ \\
\hline Aluminium (Al) & 10 & 0 & G2alt 10 \\
\hline Lead $(\mathrm{Pb})$ & 7 & 2 & zntA/yhho 4 ; zraS/hydG 3 ; pbrA 2 \\
\hline Bismuth (Bi) & 0 & 8 & $\operatorname{arsR} 8$ \\
\hline Vanadium (V) & 8 & 0 & yieF 6 ; mexl 2 \\
\hline Cobalt (Cobalt) & 2 & 0 & $\mathrm{mdrL} / \mathrm{yfmO} 2$ \\
\hline Gold (Au) & 1 & 0 & gesB 1 \\
\hline Gallium (Ga) & 1 & 0 & fptA 1 \\
\hline
\end{tabular}

